\title{
Zur Anfechtbarkeit verwaltungsorganisatorischer Anordnungen
}

Kommentar BGE 1C_517/2016 vom 12. April 2017 (Urteil «Cazis»)

Bernhard Waldmann / Gregor Bachmann *

In einem neuen Leitentscheid äussert sich das Bundesgericht zur Frage, ob gegen die Verlegung einer Kehrichtsammelstelle gestützt auf die Rechtsweggarantie (Art. 29a BV) der Weg an eine richterliche Behörde offenstehen muss. Das Urteil trägt zum einen zur Klärung der Rechtsnatur der verwaltungsorganisatorischen Anordnungen bei. Zum anderen präzisiert es den Begriff der «schützenswerten Rechtsposition» und mithin den Anwendungsbereich der Rechtsweggarantie («Rechtsstreitigkeiten»). Der nachfolgende Beitrag fasst das Urteil zusammen und versucht eine dogmatische Einordnung.

I. Sachverhalt und Prozessgeschichte .............................................................. 92

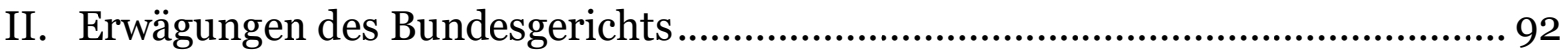

1. Anspruch auf eine anfechtbare Verfügung/Rechtsverweigerung ................... 93

2. Verletzung der Rechtsweggarantie....................................................... 93

a) Rechtsnatur und Voraussetzungen der Anfechtbarkeit ............................... 94

b) Anwendungsfälle der «schützenswerten Rechtsposition» ........................... 94

c) Beurteilung im vorliegenden Fall ............................................................ 95

d) Rückweisung zur materiellen Entscheidung ............................................ 96

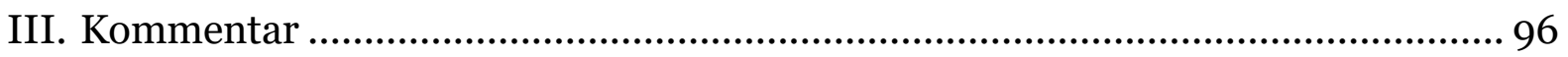

1. Begriff der verwaltungsorganisatorischen Anordnung ............................... 96

2. Rechtsschutz bei organisatorischen Anordnungen ................................... 97

3. Anwendungsbereich von Art. 29a BV ...................................................... 99

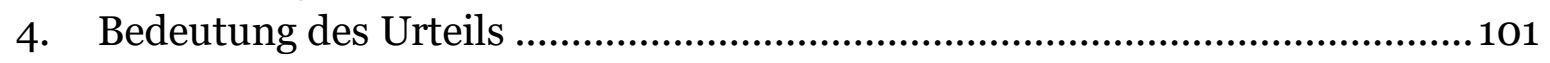

Zitiervorschlag: Bernhard Waldmann / Gregor Bachmann, Zur Anfechtbarkeit verwaltungsorganisatorischer Anordnungen, in: sui-generis 2017, S. 91

URL: $\quad$ sui-generis.ch/37

DOI: $\quad$ https://doi.org/10.21257/sg.37

* Prof. Dr. Bernhard Waldmann ist ordentlicher Professor für Staats- und Verwaltungsrecht an der Universität Freiburg i.Ue. sowie Direktor am Institut für Föderalismus.

Gregor Bachmann, MLaw, ist wissenschaftlicher Assistent am Institut für Föderalismus. 


\section{Sachverhalt und Prozessgeschichte}

1 Die Gemeinde Cazis liegt in der Region Viamala im Kanton Graubünden und verfügt über fünf örtlich getrennte Gemeindefraktionen (Cazis, Präz, Tartar, Sarn und Portein). Am 18. Dezember 2015 teilte die Gemeinde Cazis den Einwohnern und Ferienhausbesitzern der Fraktion Portein ihren Beschluss mit, die Annahmemöglichkeit für Hauskehricht beim Kehrichthäuschen Portein auf den 6. Januar 2016 einzustellen. Fortan könnten die Anwohner den Hauskehricht bei der Sammelstelle in der Fraktion Sarn abgeben. Gemeinde Cazis erhoben B. und weitere Mitunterzeichner (unter anderem A.) bei der Gemeinde Einspruch. Mit Schreiben vom 26. Januar 2016 teilte die Gemeinde den Einsprechern mit, an ihrem Beschluss festzuhalten. Sie wies weiter darauf hin, dass es sich dabei um einen reinen Verwaltungsentscheid handle, gegen den keine Einsprachemöglichkeit bestehe. Im März und April 2016 ersuchten A. und B. die Gemeinde Cazis in separaten Schreiben um Zustellung eines anfechtbaren Entscheids. Die Gemeinde wiederholte daraufhin in ihren Schreiben vom 21. März und 22. April 2016 ihren Standpunkt, dass es sich bei der Schliessung der Sammelstelle um einen «reinen Verwaltungsentscheid» ohne Einsprachemöglichkeit handle.

3 Am 3. bzw. 18. Mai 2016 erhoben A. und B. Beschwerde beim Verwaltungsgericht des Kantons Graubünden und stellten folgende Rechtsbegehren:

1. Es sei festzustellen, dass die Beschwerdegegnerin Rechtsverweigerung begeht.
2. Es sei die Beschwerdegegnerin anzuweisen, den Beschwerdeführern unverzüglich eine Verfügung betreffend die Aufhebung der Kehrichtsammelstelle in Portein zu eröffnen.

3. Eventualiter sei die Beschwerdegegnerin zu verpflichten, die Eingabe vom 31. Dezember 2015 als Beschwerde gegen einen Realakt entgegenzunehmen.

4 Das Verwaltungsgericht wies die Beschwerde am 16. August 2016 ab, auferlegte den Beschwerdeführern die Gerichtskosten jedoch nur zu 4/5 und sprach ihnen eine Parteientschädigung in der Höhe von 1/5 ihrer Aufwendungen zu. Am 8. November 2016 erhoben A. und B. gegen den Entscheid des Verwaltungsgerichts Beschwerde in öffentlich-rechtlichen Angelegenheiten an das Bundesgericht.

\section{Erwägungen des Bundesgerichts}

$5 \mathrm{Zu}$ den Eintretensvoraussetzungen äussert sich das Bundesgericht nur kurz (vgl. E. 1.). Ausgehend vom Interesse der Beschwerdeführer an der Öffnung des Rechtsweges gegen die Sammelstellenschliessung erachtet das Bundesgericht das Interesse der Beschwerdeführer an der Aufhebung oder Änderung des angefochtenen Entscheids des Verwaltungsgerichts Graubünden als schutzwürdig i.S.v. Art. 89 Abs. $1 \mathrm{BGG}^{1}$ und tritt in der Folge auf die Beschwerde ein. Ob es sich beim vorinstanzlich angefochtenen Akt (dem Entscheid der Gemeinde über die Schliessung der Sammelstelle) um eine Verfügung oder einen Realakt handelte, spiele im Zusammenhang mit dem Eintreten keine Rolle, sondern sei allenfalls

Bundesgesetz vom 17. Juni 2005 über das Bundesgericht (BGG; SR 173.110). 
für die Frage relevant, ob das Verwaltungsgericht die Anfechtbarkeit der Sammelstellenschliessung zu Unrecht verneint habe.

6 In «materieller» Hinsicht hatte sich das Bundesgericht mit zwei Fragen auseinanderzusetzen. Zum einen, ob die Gemeinde Cazis über die Schliessung der Sammelstelle eine Verfügung hätte erlassen müssen und insofern eine Rechtsverweigerung begangen hatte. Zum anderen, ob die Rechtsweggarantie (Art. 29a $\mathrm{BV}^{2}$ ) im vorliegenden Fall einen Anspruch auf gerichtliche Beurteilung einräumt.

\section{Anspruch auf eine anfechtbare Ver- fügung/Rechtsverweigerung}

7 Die Beschwerdeführer machen geltend, das Verwaltungsgericht habe den Anspruch auf rechtliches Gehör (Art. 29 Abs. 2 BV) verletzt, indem es sich mit ihrem Antrag auf Feststellung einer Rechtsverweigerung nicht auseinandergesetzt habe. Eine Rechtsverweigerung der Gemeinde sehen sie in deren Weigerung, über die Schliessung der Sammelstelle in der Fraktion Portein eine anfechtbare Verfügung zu erlassen (E. 2.1.). Das Bundesgericht hält dazu fest, dass das Verwaltungsgericht den Antrag geprüft, jedoch verworfen habe, da nach Art. 28 Abs. 4 und 49 Abs. 3 VRG/GR3 unmittelbar Beschwerde gegen Realakte, die in Rechte oder Pflichten von Personen eingreifen, geführt werden könne. Damit habe die Gemeinde keine Pflicht zum Erlass einer Verfügung getroffen,

\footnotetext{
Bundesverfassung vom 18. April 1999 der Schweizerischen Eidgenossenschaft (BV; SR 101).

3 (Bündnerisches) Gesetz vom 31. August 2006 über die Verwaltungsrechtspflege (VRG; BR 370.100).
}

weshalb auch keine Rechtsverweigerung vorliege.

8 Bezüglich der Weiterleitung des Einspruchs der Beschwerdeführer an das Verwaltungsgericht habe die Gemeinde jedoch eine Rechtsverweigerung begangen, wie das Verwaltungsgericht zutreffend festgehalten habe (E. 2.2.). Der Einspruch vom 31. Dezember 2015 hätte als Beschwerde gegen einen Realakt entgegengenommen und gemäss Art. 4 Abs. 3 VRG/GR zuständigkeitshalber an das Verwaltungsgericht weitergeleitet werden müssen. Diese Verfahrensverletzung war nach Auffassung des Verwaltungsgerichts jedoch nicht entscheidrelevant, da auf die Beschwerde auch bei rechtzeitiger Weiterleitung durch die Gemeinde nicht hätte eingetreten werden können. Das Verwaltungsgericht hatte die Rechtsverweigerung deshalb lediglich im Rahmen der Kostenverteilung berücksichtigt und verzichtete auf die formelle Feststellung der Rechtsverweigerung. Das Bundesgericht stützt diese Auffassung und weist den Antrag der förmlichen Feststellung der Rechtsverweigerung ebenfalls ab (E. 2.3.).

\section{Verletzung der Rechtsweggarantie}

9 Anschliessend prüft das Bundesgericht die Frage, ob das Verwaltungsgericht die Anfechtbarkeit der Sammelstellenschliessung verneinen durfte. Nach der Darlegung der Standpunkte der Beschwerdeführer, der Vorinstanz und der Gemeinde (E. 3.) geht das Bundesgericht auf die Frage ein, ob die Rechtsweggarantie nach Art. 29a BV einen Anspruch auf gerichtliche Überprüfung der Sammelstellenschliessung in der Fraktion Portein einräumt (E. 4.). Gemäss Art. $29 a$ BV hat jede Person bei Rechtsstreitigkei- 
ten Anspruch auf Beurteilung durch eine richterliche Behörde. In Ausnahmefällen können Bund und Kantone die richterliche Beurteilung durch Gesetz ausschliessen (Art. 29a BV Satz 2). Im vorliegenden Verfahren war insbesondere zu beurteilen, ob es sich bei der Streitigkeit über die Schliessung der Sammelstelle um eine «Rechtsstreitigkeit» i.S.v. Art. $29 a$ BV handelt.

\section{a) Rechtsnatur und Voraussetzun- gen der Anfechtbarkeit}

Ausgehend von Lehre und Rechtsprechung zum Anwendungsbereich von Art. 29a BV (E. 4.1) äussert sich das Bundesgericht zunächst zur Rechtsnatur der Schliessung der Sammelstelle für Hauskehricht in der Fraktion Portein (E. 4.2.). Das Bundesgericht qualifiziert die Schliessung der Sammelstelle implizit als verwaltungsorganisatorische Anordnung und hält fest, dass verwaltungsorganisatorische Anordnungen grundsätzlich nicht darauf gerichtet sind, unmittelbar Rechte und Pflichten von Bürgern zu begründen oder zu ändern. Aus diesem Grund ergingen sie nicht in Verfügungsform und es bestünde in der Regel selbst dann keine Rechtsschutzmöglichkeit, wenn eine Massnahme mittelbare Auswirkungen auf Private hat, wie beispielsweise die Umbenennung einer Strasse (E. 4.2., mit Hinweisen). Eine Anfechtungsmöglichkeit müsse jedoch nach Art. 29a BV eröffnet werden, «wenn die Anordnung geeignet ist, die Position einer Person als Träger von Rechten und Pflichten gegenüber dem Staat zu beeinflussen» (E. 4.2. [Hervorhebung nur hier]). Das Bundesgericht bezieht sich dabei auf die Urteile zur Versetzung eines Polizeifunktionärs im Kanton Genf (BGE 136 I 323) sowie zur Störfallvorsorge im
Atomkraftwerk Mühleberg (BGE 140 II 315). In letzterem wurde entschieden, dass eine Berufung auf die Rechtsweggarantie neben einem schutzwürdigen Interesse auch ein «Berührtsein in Rechten und Pflichten» - eine schützenswerte Rechtsposition - voraussetzt (E. 4.2., mit Verweis auf BGE 140 II 315, E. 4.5). Im Sinne einer Minimalgarantie fordere Art. 29a BV, dass Rechtsschutz gewährt wird, wenn ein Realakt oder eine verwaltungsinterne Anordnung individuelle, schützenswerte Rechtspositionen berührt. Dabei stehe es den Kantonen frei, in ihrem Zuständigkeitsbereich eine andere Konzeption des Rechtsschutzes gegen verfügungsfreies staatliches Handeln vorzusehen als der Bundesgesetzgeber in Art. 25a VwVG4.

\section{b) Anwendungsfälle der «schüt- zenswerten Rechtsposition»}

11 Schützenswerte Rechtspositionen können sich laut Bundesgericht aus dem Verfassungs-, Gesetzes- oder Verordnungsrecht in allen Rechtsbereichen ergeben (E. 4.3., mit Verweis auf BGE 136 I 323, E. 4.3). Das Bundesgericht nennt anschliessend zwei Konstellationen: Zum einen bestehe eine schützenswerte Rechtsposition jedenfalls dann, «wenn in vertretbarer Weise geltend gemacht wird, es bestehe ein Anspruch auf ein bestimmtes staatliches Handeln oder Unterlassen, der durch den angefochtenen Akt verletzt werde» (E. 4.3.1. [Hervorhebung nur hier], mit Verweis u.a. auf BGE 140 II 315, E. 4.6-4.8). Zum anderen könne eine schützenswerte Rechtsposition auch hinsichtlich der Modalitäten der Rechtsausübung bestehen (E. 4.3.2.). Das

4 Bundesgesetz vom 20. Dezember 1968 über das Verwaltungsverfahren (VwVG; SR 172.021). 
Bundesgericht nimmt dabei Bezug auf zwei unpublizierte Urteile (E. 4.3.2. und 4.3.3.):

Im Urteil 2P.324/2001 vom 28. März 2002 ging es um einen Erstklässler, der einem 500 Meter weiter entfernten Schulhaus zugewiesen worden war. Das Bundesgericht entschied, dass ihm bzw. seinen Eltern zwecks Überprüfung der Rechtmässigkeit und Zumutbarkeit der Massnahme der Rechtsweg eröffnet werden müsse, da die Anordnung in erheblicher Weise in das Leben und den Tagesablauf des betroffenen Kindes eingreife (a.a.O., E. 3.4).

Im Urteil 2P.12/2001 vom 25. Juli 2001 ging es wie im vorliegenden Fall um eine Reorganisation der Kehrichtabfuhr. Die Zürcher Gemeinde Hombrechtikon hatte beschlossen, den Hauskehricht an einer bestimmten, ausserhalb der Bauzone gelegenen Strasse nicht mehr abzuholen, sondern in 350 Metern Entfernung einen Sammelcontainer einzurichten. Der Beschluss war von der Gemeinde als Verfügung bezeichnet worden, was auch von den nachfolgenden Instanzen nicht in Zweifel gezogen worden war. Das Bundesgericht trat auf die staatsrechtliche Beschwerde ein, weil die Beschwerdeführer als betroffene Benützer der kommunalen Kehrichtentsorgung durch den angefochtenen Entscheid in rechtlich geschützten Interessen berührt seien (a.a.O., E. 1b). Die Vollziehungsverordnung der Gemeinde Hombrechtikon sah vor, dass der Hauskehricht in der Nähe der Liegenschaft deponiert werden könne und knüpfte die Bereitstellung an einem weiter entfernten Ort an bestimmte Voraussetzungen (vgl. a.a.O., E. 2d). Das Verwaltungsgericht hatte die modifizierte Kehrichttour als materielle Änderung dieser Vollziehungsbestimmungen qualifiziert, weshalb die Beschwerdeführer eine Verschlechterung ihrer Rechtsposition geltend machen konnten (a.a.O., E. 2e).

\section{c) Beurteilung im vorliegenden Fall}

12 In E. 4.4. beurteilt das Bundesgericht die Entsorgungspflicht in Cazis unter dem Aspekt des Begriffs der «schützenswerten Rechtsposition»5. In der Gemeinde Cazis bestehe, anders als im vom Bundesgericht entschiedenen Fall der Gemeinde Hombrechtikon, kein Anspruch auf eine Sammelstelle innert einer gewissen Distanz oder innerhalb der Gemeindefraktion. Die Beschwerdeführer seien jedoch gleichwohl nach Art. $31 b$ Abs. 3 USG $^{6}$ i.V.m. Art. 12 des kommunalen Abfallgesetzes verpflichtet, den Hauskehricht einer Sammelstelle in der Gemeinde Cazis zu übergeben. $\mathrm{Zu}$ den Modalitäten der Entsorgungspflicht der Anwohner hält das Bundesgericht fest: Zwar stehe den Gemeinden bei der Ausgestaltung der Entsorgungspflicht ein erheblicher Spielraum zu, doch müssten den Anwohnern Sammelstellen in genügender Anzahl, Dichte und Frequenz angeboten werden, die angemessen situiert seien, wobei aber kein Anspruch auf die bequemste Lösung bestehe (E. 4.4., mit Hinweisen).

13 Die Entsorgungspflicht werde vorliegend insofern berührt, «als Einwohner und Ferienhausbesitzer in Portein ihren Abfall künftig $\mathrm{zu}$ einer anderen, weiter entfernt gelegenen Sammelstelle in einer anderen Gemeindefraktion bringen müssen». Der Rechtsweg müsse bei dieser Rechtslage offenstehen, «wenn Privatpersonen plausibel geltend machen, dass ihren gerechtfertigten Bedürfnissen bei der Neuorganisation der Kehrichtabfuhr

$5 \longdiv { \text { Synonym dazu verwendet das Bundesgericht } }$ auch den Begriff der «schutzwürdigen Rechtsposition».

6 Bundesgesetz vom 7. Oktober 1983 über den Umweltschutz (USG; SR 814.01). 
nicht Rechnung getragen worden sei und die neuen bzw. verbleibenden Sammelstellen für sie unzumutbar oder jedenfalls mit erheblichen Nachteilen verbunden seien, das heisst die Erfüllung ihrer gesetzlichen Verpflichtung zur Ablieferung der Siedlungsabfälle erheblich erschwert werde». Die grössere Bequemlichkeit der bisherigen Lösung genüge dazu allerdings noch nicht. Vorliegend machten die Beschwerdeführer geltend, die in $1.6 \mathrm{~km}$ Entfernung liegende nächstgelegene Sammelstelle erschwere die Entsorgung; insbesondere sei es nicht mehr zumutbar, den Abfall zu Fuss zur Sammelstelle zu bringen. Das Bundesgericht lässt dies zur Annahme einer schützenswerten Rechtsposition genügen: «Damit machen sie [die Beschwerdeführer] in genügender Weise geltend, die strittige Aufhebung der Sammelstelle berühre ihre Pflicht zur gesetzeskonformen Entsorgung ihres Hauskehrichts bzw. ihren Anspruch, von der Gemeinde eine zumutbare Sammelstelle zur Verfügung gestellt zu erhalten» (E. 4.4.). Es liege daher eine Rechtsstreitigkeit i.S.v. Art. 29a BV vor, weshalb der Zugang zu gerichtlichem Rechtsschutz zu gewähren sei.

\section{d) Rückweisung zur materiellen Entscheidung}

Bundesgericht weist die Sache in der Folge - bei Erfüllung der weiteren Eintretensvoraussetzungen - zur materiellen Entscheidung an das Verwaltungsgericht zurück (E. 5.). Eine Rückweisung an die Gemeinde Cazis erübrige sich aufgrund der direkten Anfechtbarkeit von Realakten beim Verwaltungsgericht im Kanton Graubünden.

\section{Kommentar}

\section{Begriff der verwaltungsorganisato- rischen Anordnung}

15 Zur Rechtsnatur des in Frage stehenden Verwaltungsaktes - der Schliessung einer Kehrichtsammelstelle - äussert sich das Bundesgericht im (zur amtlichen Publikation vorgesehenen) Urteil Cazis nicht explizit. Die abstrakten Ausführungen des Bundesgerichts zu den verwaltungsorganisatorischen Anordnungen lassen aber darauf schliessen, dass auch der vorliegend in Frage stehende Akt zu diesen gezählt wird7. Verwaltungsorganisatorische Anordnungen gehören zu einer Kategorie von Handlungsformen, die dogmatisch erst wenig durchdrungen ist: Den Verwaltungsinnenakten. Diese können sowohl Rechtsakte (= Verwaltungsakte, die unmittelbar auf einen rechtlichen Erfolg ausgerichtet sind) als auch Realakte (= Verwaltungsakte, die primär einen tatsächlichen Erfolg herbeiführen wollen) umfassen ${ }^{8}$. Verwaltungsakte, die auf einen Rechtserfolg im Aussenverhältnis ausgerichtet sind, erfüllen den Tatbestand der Verfügung 9 . Daneben können auch Innenakte Rechtsakte sein, ohne dass sie deswegen als Verfügungen zu qualifizieren wären. Die Qualifikation als Verfügung hat das Bundesgericht vorliegend im Übrigen klar abgelehnt, indem es den Beschwerdeantrag auf Feststellung einer Rechtsverweigerung, die von den Beschwerdeführern in der Verweigerung einer anfechtbaren Verfügung

\footnotetext{
Urteil Cazis, E. 4.2.

8 Bernhard Waldmann, Vom Umgang mit organisatorischen, innerdienstlichen und anderen Anordnungen ohne Verfügungscharakter, in: ZSR 2014 I, S. 489-515, S. 490 f.

9 BGE 136 I 323 E. 4.4 (Versetzung); vgl. dazu Waldmann (Fn. 8), S. 495 (mit weiteren Hinweisen).
} 
erblickt wurde, abgewiesen hat ${ }^{10}$. Die Abgrenzung zwischen Rechts- und Realakten bereitet im Einzelfall jedoch oft Mühe, da die Zielsetzung eines Verwaltungsaktes nur selten eindeutig bestimmbar ist ${ }^{11}$.

Die Schliessung der Kehrichtsammelstelle in der Fraktion Portein steht im $\mathrm{Zu}$ sammenhang mit der Reorganisation der Kehrichtabfuhr in der Gemeinde Cazis. Es geht damit um eine Anordnung, welche die Organisation und den Betrieb einer durch die Verwaltung erbrachten Dienstleistung (= Kehrichtabfuhr) betrifft. Mit ihr werden die Modalitäten, nach welchen die Verwaltung eine Dienstleistung anbietet, neu festgelegt ${ }^{12}$. Als verwaltungsorganisatorische Anordnung ist sie auf die Ordnung der tatsächlichen Verhältnisse innerhalb der Verwaltung, i.c. die Organisation der Kehrichtentsorgung, ausgerichtet. Die Regelung von Rechten und Pflichten der Einwohnerinnen und Einwohner der Fraktion Portein steht nicht im Vordergrund ${ }^{13}$. Damit kann die Schliessung der Kehrichtsammelstelle als Innen-Realakt (mit Aussenwirkungen) qualifiziert werden. $\mathrm{Zu}$ den verwaltungsorganisatorischen Anordnungen, die auf die Modalitäten der Erbringung einer Dienstleistung durch die Verwaltung gerichtet sind, zählen beispielsweise auch die Festsetzung

10 Urteil Cazis, E. 2.

11 Vgl. dazu Markus Müller, Rechtsschutz gegen Verwaltungsrealakte, in: Pierre Tschannen (Hrsg.), Berner Tage für die juristische Praxis BTJP 2006, Neue Bundesrechtspflege, Auswirkungen der Totalrevision auf den kantonalen und eidgenössischen Rechtsschutz, Bern 2007, S. $313-373$, S. 320.

12 Vgl. zum Ganzen Waldmann (Fn. 8), S. 496.

13 Vgl. Urteil Cazis, E. 4.2: «Verwaltungsorganisatorische Anordnungen sind nicht darauf gerichtet, unmittelbar Rechte und Pflichten von Bürgern zu begründen oder zu ändern.» von Fahrplänen und Linienführungen der öffentlichen Verkehrsbetriebe ${ }^{14}$ sowie die Festlegung von Schalteröffnungszeiten von Behörden ${ }^{15}$.

17 Mit verwaltungsorganisatorischen Anordnungen sind grundsätzlich nur reflexweise Rechtswirkungen verbunden, in erster Linie sind derlei Anordnungen auf einen tatsächlichen Erfolg ausgerichtet. Es ist jedoch nicht ausgeschlossen, dass es im Anschluss an verwaltungsorganisatorische Anordnungen zum Erlass von Innen-Rechtsakten (v.a. Dienstbefehle ${ }^{16}$ ) oder auch Verfügungen (z.B. Entlassungen nach der Schliessung einer Schule) kommt ${ }^{17}$.

\section{Rechtsschutz bei organisatorischen Anordnungen}

18 Ausgehend von der Theorie des subjektiven Rechts und der damit einhergehenden Trennung von staatlicher Sphäre und Aussensphäre wurden Innenakte (wie auch das objektive Recht) lange nicht als rechtsschutzwürdig betrachtet ${ }^{18}$. Mit der zunehmenden Orientierung der Verwaltungsrechtspflege am Rechtsschutzinteresse der Betroffenen, was nicht zuletzt auf die Verfahrensgarantien der EMRK

$14 \overline{\text { Vgl. Entscheid des Bundesrates VPB } 58.79 \text { vom } 1 .}$ Januar 1993, E. 4 (Fahrplan); Entscheid des Bundesrates VPB 44.42 vom 5. Oktober 1979, E. II.1 und Entscheid des Bundesrates VPB 50.51 vom 16. Oktober 1985, E. 2 (Linienführung Postautokurs); Entscheid des Bundesrates VPB 60.20 vom 26. April 1995, E. 5 (Umstellung von Bahnauf Busbetrieb); Anders noch Entscheid des Bundesrates VPB 47.51 vom 19. Oktober 1983, E. II.1 (Aufhebung Direktverbindung); Entscheid des Bundesrates VPB 45.17 vom 14. Januar 1981 (Aufhebung Haltestelle für Schnellzug).

15 Vgl. Entscheid des Eidg. Finanzdepartementes (EFD) VPB 53.38 vom 28.11.1988, E. 2 (Abfertigungszeiten Zollamt).

16 Vgl. dazu Waldmann (Fn. 8), S. 499 f.

17 Vgl. dazu Waldmann (Fn. 8), S. $497 \mathrm{f}$.

18 Müller (Fn. 11), S. 370. 
zurückzuführen ist, konnte diese Sichtweise jedoch nicht aufrechterhalten werden ${ }^{19}$. Nach heutiger Auffassung besteht auch bei Verwaltungsakten, welche die Elemente des materiellen Verfügungsbegriffs nicht erfüllen, Anspruch auf $\mathrm{Zu}-$ gang zum Rechtsschutz, soweit schützenswerte Rechtspositionen von Privaten berührt werden. Angesprochen sind damit zum einen Verwaltungsrealakte, zum anderen aber auch Verwaltungsrechtsakte, welche - wie insbesondere InnenRechtsakte - keine Verfügungen sind.

19 Auch verwaltungsorganisatorische Anordnungen können Private in schutzwürdigen Rechtspositionen berühren. Für Rechtsstreitigkeiten, die aus verwaltungsorganisatorischen Anordnungen hervorgehen, muss deshalb gemäss Art. 29a BV Zugang zu wirksamem gerichtlichem Rechtsschutz gewährt werden $^{20}$. Die Rechtsweggarantie von Art. 29a BV verlangt dabei keine Ausweitung des Verfügungsbegriffs ${ }^{21}$; die Kan-

19 Müller(Fn. 11), S. 334 f.

20 Urteil Cazis, E. 4.2; Waldmann (Fn. 8), S. 498; a.M. Andreas Kley, in: Bernhard Ehrenzeller/ Benjamin Schindler/Rainer J. Schweizer/Klaus A. Vallender (Hrsg.), Die schweizerische Bundesverfassung, St. Galler Kommentar, 3. Aufl., Zürich/St. Gallen 2014, Art. $29 a$ N 11.

21 Vgl. auch René Wiederkehr, Öffentliches Verfahrensrecht, Bern 2016, Rz. 246; so auch bereits Bernhard Waldmann, Justizreform und öffentliche Rechtspflege - quo vadis?, in: AJP 7/2003, S. 747-760, S. 750; Anders, u.E. unzutreffend Urteil des Bundesgerichts 2C_272/2012 vom 9. Juli 2012 E. 4.3 (Turnlektionen); ihm folgend Regina Kiener/Bernhard Rütsche/Mathias Kuhn, Öffentliches Verfahrensrecht, 2. Aufl., Zürich/St. Gallen 2015, Rz. 361-366; Für eine Neudefinition des Anfechtungsobjekts der Verwaltungsrechtspflege ausgehend vom Rechtsschutzinteresse spricht sich auch Markus Müller, (Schleich-)Wege zum Verwaltungsrechtsschutz, Plädoyer für eine praxistaugliche Dogmatik und eine dogmatiktreue Praxis, in: ZBl 2/2015, S. 59-76, S. 75 sowie Ders., Kommentar zu BGE 141 II 233, in: ZBl 8/2015, S. 442-449, S. 446-449, aus. tone sind vielmehr frei in der Wahl ihrer Lösungen ${ }^{22}$. Auf Bundesebene wurde mit Art. 25a VwVG die Möglichkeit geschaffen, eine Verfügung über Realakte zu verlangen. Zwölf Kantone haben in der Zwischenzeit analoge Regelungen in ihren Verfahrensgesetzen getroffen (BaselStadt, Genf, Luzern, Nidwalden, Obwalden, Schaff-hausen, Schwyz, Solothurn, Tessin, Uri, Zug, Zürich)23. Weitere zwölf Kantone kennen keine spezifischen Verfahrensregeln in Bezug auf Realakte (Aargau, Appenzell-Ausserrhoden, Appenzell-Innerrhoden, Basel-Landschaft, Bern, Glarus, Jura, Neuenburg, St. Gallen, Thurgau, Waadt, Wallis). Der Kanton Freiburg lässt die Beschwerde an das Kantonsgericht immer dann $\mathrm{zu}$, wenn das Bundesrecht einen entsprechenden Anspruch einräumt 24 .

22 Urteil Cazis, E. 4.2; Waldmann(Fn. 8), S. 508.

23 Basel-Stadt: $\S 38$ a des Gesetzes betreffend die Organisation des Regierungsrates und der Verwaltung des Kantons Basel-Stadt vom 22. April 1976 (OG; SG 153.100); Genf: Art. 4A der Loi sur la procédure administrative vom 12. September 1985 (LPA; RSG E 5 10); Luzern: § 44a des Gesetzes über die Verwaltungsrechtspflege vom 3 . Juli 1972 (VRG; SRL 40); Nidwalden: Art. 6oc des Gesetzes über das Verwaltungsverfahren und die Verwaltungsrechtspflege vom 8. Februar 1985 (VRG; NG 265.1); Obwalden: Art. 2a der Verordnung über das Verwaltungs- und Verwaltungsbeschwerdeverfahren vom 29. Januar 1998 (VRPV; GDB 133.21); Schaffhausen: Art. 7a des Gesetzes über den Rechtsschutz in Verwaltungssachen vom 20. September 1971 (VRG; SHR 172.200); Schwyz: § 34a des Verwaltungsrechtspflegegesetzes vom 6. Juni 1974 (VRP; SRSZ 234.110); Solothurn: §28bis des Gesetzes über den Rechtsschutz in Verwaltungssachen vom 15. November 1970 (VRG; BGS 124.11); Tessin: Art. 64 der Legge sulla procedura amministrativa vom 24. September 2013 (LPAmm; RL/TI 3.3.1.1); Uri: Art. 25a der Verordnung über die Verwaltungsrechtspflege vom 23. März 1994 (VRPV; RB 2.2345); Zug: § 21a des Gesetzes über den Rechtsschutz in Verwaltungssachen vom 1. April 1976 (VRG; BGS 162.1); Zürich: § 10c des Verwaltungsrechtspflegegesetzes vom 24. Mai 1959 (VRG; LS175.2).

24 Art. 7a und Art. 114 Abs. 2 lit. b des (freiburgi- 
20 Eine besondere Lösung kennt der (im vorliegenden Fall betroffene) Kanton Graubünden, welcher die direkte Anfechtung von Realakten beim Verwaltungsgericht zulässt. Dazu bedient sich der Kanton einer Verfügungsfiktion und stellt «Realakte, die in Rechte und Pflichten von Personen eingreifen», genauso wie Rechtsverweigerung und Rechtsverzögerung Verfügungen («Entscheiden») gleich ${ }^{25}$. Diese Lösung - die Ausweitung des Anfechtungsobjekts der Verwaltungsrechtspflege - wird von der Lehre kritisch betrachtet, da der mit der Beschwerde betrauten richterlichen Behörde das «Dossier» fehlt, weshalb sie nicht selten aufwändig den Sachverhalt abklären muss ${ }^{26}$. Gerade diesbezüglich erweist sich ein Verwaltungsverfahren bei der Instanz, welche für den Realakt zuständig ist, als effizienter ${ }^{27}$.

Im vorliegenden Fall führte die Bündner Lösung im Übrigen zu einiger Konfusion. Die Beschwerdeführer hielten bis zum Verfahren vor Bundesgericht an ihrem Antrag fest, es sei eine Rechtsverweige-

schen) Gesetzes über die Verwaltungsrechtspflege vom 23. Mai 1991 (VRG; SGF 150.1).

25 Art. 28 Abs. 4 und Art. 49 Abs. 3 VRG/GR: «Als Entscheide gelten auch Rechtsverweigerung und Rechtsverzögerung sowie Realakte, die in Rechte und Pflichten von Personen eingreifen.»

26 Pierre Tschannen, Amtliche Warnungen und Empfehlungen, in: ZSR 1999 II, S. 353-455, Rz. 149; Alfred Kölz/Isabelle Häner/Martin Bertschi, Verwaltungsverfahren und Verwaltungsrechtspflege des Bundes, 3. Aufl., Zürich/Basel/ Genf 2013, Rz. 332; Waldmann (Fn. 8), S. 511; a.M. Müller (Fn. 11), S. 369, welcher zwar anerkennt, dass das fehlende Dossier «nicht ideal» sei, jedoch darauf hinweist, dass heute schon Rechtsmittelinstanzen ab und an versäumte Instruktionsmassnahmen nachholten. Darüber hinaus könne diesem Problem mit der Vorschaltung eines Einspracheverfahrens begegnet werden (S. 369 und 373).

27 Tschannen (Fn. 26), Rz. 149; vgl. auch Müller (Fn. 11), S. 369. rung seitens der Gemeinde festzustellen, da diese ihnen den Erlass einer Verfügung verwehrt habe. Die direkte Anfechtung von Realakten scheint in der Beschwerde an das Verwaltungsgericht nicht im Vordergrund gestanden zu haben; vielmehr ging man über das Instrument der Rechtsverweigerungsbeschwerde vor. Das Bündner Verwaltungsgericht nahm die Angelegenheit anschliessend als Beschwerde gegen einen Realakt entgegen, stellte aber immerhin eine Rechtsverweigerung der Gemeinde bezüglich der nicht erfolgten Weiterleitung des «Einspruchs» an das Verwaltungsgericht fest. Das Bundesgericht stützt das Vorgehen des Bündner Verwaltungsgerichts in diesem Punkt ${ }^{28}$.

\section{Anwendungsbereich von Art. 29a BV}

22 Der Begriff der «Rechtsstreitigkeiten» von Art. 29a BV setzt, gleich wie die Voraussetzung des «Berührtseins in Rechten und Pflichten» von Art. 25a VwVG, eine schützenswerte Rechtsposition voraus ${ }^{29}$. Ihre Grundlage können schützenswerte Rechtspositionen im Verfassungs-, Gesetzes-, aber auch im Verordnungsrecht aller Rechtsbereiche finden ${ }^{30}$. Bisher ging man davon aus, zur Begründung einer schützenswerten Rechtsposition müsse dargelegt werden, «es bestehe ein Anspruch auf ein bestimmtes staatliches Handeln oder Unterlassen, der durch den angefochtenen Akt verletzt werde.»31 Insbesondere im wegweisen-

28 Urteil Cazis, E. 2.

29 Urteil Cazis, E. 4.2; BGE 140 II 315 E. 4.5 (Störfallvorsorge KKW Mühleberg); vgl. auch Urteil des Bundesgerichts 2C_272/2012 vom 9. Juli 2012 E. 4.3. (Turnlektion).

30 Urteil Cazis, E. 4.3, mit Verweis auf BGE 136 I 323 E. 4.3 (Versetzung).

31 Urteil Cazis, E. 4.3.1, mit Verweis auf BGE 140 II 315 E. 4.6-4.8 (Störfallvorsorge KKW Mühle- 
den Urteil zur Störfallvorsorge im KKW Mühleberg aus dem Jahr 2014 (BGE 140 II 315) wurde eine schützenswerte Rechtsposition aus der Schutzpflicht des Staates vor Nuklearunfällen, die sich aus den Grundrechten auf Leben (Art. 10 Abs. 1 BV) und auf persönliche Freiheit (Art. 10 Abs. 2 BV) sowie der diese konkretisierenden Kernenergiegesetzgebung ergibt, und den sich daraus ergebenden Ansprüchen der Privaten abgeleitet 32 . Daran anknüpfend erweitert das Bundesgericht nun aber im vorliegenden Urteil den Begriff der schützenswerten Rechtsposition auf die «Modalitäten der Rechtsausübung»33. Inwiefern damit eine neue Kategorie schützenswerter Rechtspositionen geschaffen worden ist, bleibt allerdings u.E. unklar.

Das vorliegende Urteil der ersten öffentlich-rechtlichen Abteilung lässt sich zunächst ohne dogmatische Brüche in die im Urteil der zweiten öffentlichrechtlichen Abteilung zur Störfallvorsorge im KKW Mühleberg begründete Rechtsprechung einordnen, wonach eine schützenswerte Rechtsposition aus Anspruchsnormen oder aus Verpflichtungen entstehen kann, welche auch dem Schutz der Einzelnen dienen. Vorliegend ergibt sich die schützenswerte Rechtsposition aus Art. $31 b$ USG i.V.m. Art. 35 KUSG/GR34 und Art. 12 des Abfallgesetzes der Gemeinde Cazis35. Diese Bestimmungen verpflichten einerseits die Ein-

berg) und Urteil des Bundesgerichts 2C_272/ 2012 vom 9. Juli 2012 E. 4.4.6 (Turnlektionen).

32 BGE 140 II 315 E. 4.8 (Störfallvorsorge KKW Mühleberg).

33 Urteil Cazis, E. 4.3.2.

34 (Bündnerisches) Einführungsgesetz vom 2. Dezember 2001 zum Bundesgesetz über den Umweltschutz (Kantonales Umweltschutzgesetz; BR 820.100).

35 Vgl. Urteil Cazis, E. 4.4. wohner und Eigentümer von Zweitwohnungen, ihren Kehricht in der Gemeinde $\mathrm{zu}$ entsorgen, und andererseits die Gemeinde zur Sammlung der Siedlungsabfälle. Die Aufhebung der Sammelstelle Portein berührt nicht nur die Modalitäten der Entsorgungspflicht der Einwohner und Zweitwohnungseigentümer, sondern auch die Organisation des Entsorgungsmonopols der Kantone und Gemeinden. Zwar überlassen die einschlägigen Vorschriften den Kantonen und Gemeinden für die Ausgestaltung der Entsorgung einen erheblichen Spielraum. Sie sind aber nach der Rechtsprechung zu Art. $31 b$ USG verpflichtet, zweckmässige, den gerechtfertigten Bedürfnissen des Abfalllieferanten entsprechende Entsorgungslösungen bereitzustellen ${ }^{36}$. Diese gesetzlichen Vorgaben für die Organisation des Entsorgungswesens entfalten damit auch eine Schutzwirkung zugunsten der Abfallinhaber. Schützenswerte Rechtspositionen i.S.v. Art. $29 a \mathrm{BV}$ können sich somit auch aus objektivrechtlichen Vorgaben für die Organisation und den Betrieb der durch die Verwaltung erbrachten Dienstleistungen ergeben, sofern diese Vorschriften zumindest auch dem Schutz der Privaten dienen. Schützenswerte Rechtspositionen setzen mithin keine subjektiven Rechte, so aber doch immerhin Schutznormen voraus.

24 Bei näherem Hinsehen bietet das vorliegende Urteil aber auch Raum für einen weiteren Anwendungsbereich der Rechtsweggarantie. Massgebend für die Begründung einer schützenswerten Rechtsposition wäre demnach nicht mehr primär die Frage der Schutzwirkung einer Vorschrift zugunsten der Privaten, sondern die Frage der Intensität der Nach-

36 Urteil Cazis, E. 4.4. 
teile, die sich für die Betroffenen im $\mathrm{Zu}-$ sammenhang mit den Modalitäten der Ausübung ihrer Pflichten ergeben37. Im vorliegenden Fall hatten die Beschwerdeführer vorgebracht, es sei infolge des Aufhebungsbeschlusses nicht mehr zumutbar, den Abfall in die nächstgelegene, $1.6 \mathrm{~km}$ entfernte Sammelstelle zu bringen. Damit konnten die Beschwerdeführer in genügender Weise geltend machen, die strittige Aufhebung der Sammelstelle berühre ihre Pflicht zur gesetzeskonformen Entsorgung ihres Hauskehrichts bzw. ihren Anspruch, von der Gemeinde eine zumutbare Sammelstelle zur Verfügung gestellt $\mathrm{zu}$ erhalten ${ }^{3}$. Die Beschwerdeführer haben damit einen Anspruch auf (gerichtliche) Prüfung, ob die neue Entsorgungslösung noch zweckmässig und zumutbar ist.

\section{Bedeutung des Urteils}

25 Beim Urteil Cazis handelt es sich um ein in mehrfacher Hinsicht wegweisendes Urteil. Das Bundesgericht definiert erstens die Rechtsnatur der verwaltungsorganisatorischen Anordnungen: Diese sind in aller Regel als Innen-Realakte zu qualifizieren. Rechtswirkungen stehen nicht im Vordergrund. Zweitens stellt das Bundesgericht klar, dass die Rechtsweggarantie von Art. 29a BV weder eine Ausweitung des Verfügungsbegriffs 39

37 In diese Richtung deutet auch die Auslegung von Art. 29a BV bei Kölz/Häner/Bertschi (Fn. 26), Rz. 181: «[...] Rechtsstreitigkeiten können sich auch aus Realakten der Verwaltung ergeben. Dabei geht es nicht darum, auf die Rechtsweggarantie die Theorie vom subjektiven öffentlichen Recht anzuwenden, wonach den Einzelnen durchsetzbare Rechte nur dann zukommen sollen, wenn sie sich auf eine Norm berufen können, die gerade ihrem Schutz dienen soll.»

38 Urteil Cazis, E. 4.4.

39 So noch Urteil des Bundesgerichts 2C_272/2012 vom 9. Juli 2012 E. 4.3 (Turnlektionen). Vgl. dazu oben Ziff. 2. verlangt noch den Kantonen die Übernahme des Regelungskonzepts einer «Verfügung über Realakte» analog Art. 25a VwVG aufdrängt. Die Kantone sind grundsätzlich frei, wie sie den erforderlichen Rechtsschutz sicherstellen wollen. Drittens präzisiert das Urteil den Anwendungsbereich der Rechtsweggarantie von Art. 29a BV, indem es festhält, dass sich schützenswerte Rechtspositionen auch hinsichtlich der Modalitäten der Rechtsausübung ergeben können. $\mathrm{Ob}$ damit eine Fortführung oder eine Ausweitung der an Schutznormen anknüpfenden Rechtsprechung im Bundesgerichtsurteil zur Störfallvorsorge im KKW Mühleberg (BGE 140 II 315) verbunden ist, bleibt allerdings unklar. Es scheint, als ob sich das Verständnis der beiden öffentlich-rechtlichen Abteilungen in dieser Thematik zumindest in Nuancierungen unterscheidet. 